\title{
The Equitable Edge-Coloring of Series-Parallel Graphs ${ }^{\star}$
}

\author{
Huimin Song ${ }^{1}$, Jianliang $\mathrm{Wu}^{2}$, and Guizhen $\mathrm{Liu}^{2}$ \\ ${ }^{1}$ Department of Applied Mathematics, Shandong University at Weihai, Weihai, \\ 264209, P.R. China \\ 2 School of Mathematics and System Science, Shandong University, Jinan, 250100, \\ P.R. China
}

\begin{abstract}
An edge-coloring of a graph $G$ is equitable if, for each vertex $v$ of $G$, the number of edges of any one color incident with $v$ differs from the number of edges of any other color incident with $v$ by at most one. A graph $G$ is called equitable if $G$ has an equitable edge-coloring with $k$ colors for any integer $k \geq 1$. A plane graph is series-parallel graph if it contains no subgraphs homeomorphic to $K_{4}$. In the paper, we prove that any simple and connected series-parallel graph is equitable if and only if it is not an odd circuit.
\end{abstract}

Keywords: edge-coloring; equitable, series-parallel graph.

\section{Introduction}

Throughout the paper, all graphs are finite, simple and undirected. Let $G$ be a graph, $V(G)$ and $E(G)$ denote the set of vertices and the set of edges of $G$, respectively. We associate positive integer $1,2, \ldots$ with colors, and call $C$ a $k$ edge-coloring of $G$ if $C: E \rightarrow\{1,2, \ldots, k\}$. Let $C_{v}^{-1}(i)$ denote the number of edges of $G$ incident with vertex $v$ that receive color $i$ by the coloring $C$. We call $C$ an equitable $k$-edge-coloring of $G$ if for each vertex $v \in V$

$$
\left|C_{v}^{-1}(i)-C_{v}^{-1}(j)\right| \leq 1 \quad(1 \leq i<j \leq k) .
$$

A graph $G$ is called equitable if $G$ has an equitable $k$-edge-coloring for any integer $k \geq 1$. It is an interesting problem to determine whether a graph is equitable or not. If a graph is equitable, then its edge chromatic index is equal to its maximum degree $\Delta$ and its edge cover chromatic index equal to its minimum degree $\delta$ (refer to [1] for the definition of edge cover chromatic index).

A plane graph is series-parallel graph if it contains no subgraphs homeomorphic to $K_{4}$, denoted by SP graph briefly. A series-parallel graph is a kind of very interesting graph which has many good properties and is well applied in electricity networks.

A circuit is a connected graph in which each vertex has even degree. A circuit is odd, or even, if the number of edges is odd, or even, respectively. It is stated in

\footnotetext{
^ The work is supported by NSFC(10471078,10631070, 60673059) of China.
} 
2. that a connected graph $G$ has an equitable 2-edge-coloring if and only if it is not an odd circuit. Hilton and Werra [3] gave a sufficient condition for equitable edge-coloring of simple graphs. Werra 2] has proved that all bipartite graphs are equitable. Wu Jian-liang [4] has proved that a connected outerplanar graph is equitable if and only if it is not an odd circuit.

\section{The Main Result}

In this paper, we consider the equitable edge-coloring of simple series-parallel graphs and obtain the following result.

Theorem 1. Any simple and connected series-parallel graph is equitable if and only if it is not an odd circuit.

Firstly we discuss the properties on the structure of series-parallel graphs. Wu Jian-liang has made many researchs on the structure of series-parallel graphs and gained some results by which some problems on the list edge-coloring and the linear arboricity of series-parallel graphs are successfully solved (see [5]6]). We will discuss the structure of series-parallel graphs more meticulously by new methods. Our result on the structure of series-parallel graphs will be more appropriate for the discussion of the equitable edge-coloring of series-parallel graphs.

A 2-connected series-parallel graph can be gained from $K_{2}$ by iteratively using the following operations: Replace an edge with a path of length 2 (denoted by S-operation briefly) or duplicate an edge (denoted by P-operation briefly). For every 2-connected series-parallel graph, we can express the operation process with a complete binary tree. For we always begin constructing a SP graph with $K_{2}$, let an isolated point denote the only edge in $K_{2}$ at the beginning. If an $\mathrm{S}$-operation is performed on an edge, call the point representing the edge S-node and join two son nodes to the S-node each of which represents one of the two edges on the path of length 2 respectively; If a $\mathrm{P}$-operation is performed on an edge, call the point representing the edge P-node and join two son nodes to the $\mathrm{P}$-node which represent the edge and its duplicate respectively. By the above process, every 2-connected series-parallel graph $G$ corresponds to a complete binary tree $T$.

By discussing the corresponding complete binary trees in detail, we obtain the properties on the structure of simple SP graphs which are described in the following lemma. In the following, $N_{G}(v)$ denotes the set of vertices adjacent to a vertex $v$ in $G$, without confusion, $N(v)$ briefly. A vertex $v$ is called a $k$-vertex if $\left|N_{G}(v)\right|=k$.

Lemma 1. Let $G$ be a simple and 2-connected series-parallel graph of order at least 5. Then one of the following conditions holds:

1. G has two adjacent 2 -vertices $x$ and $y$;

2. $G$ has two different 2 -vertices $x$ and $y$ and $N(x)=N(y)$;

3. $G$ has a 4-vertex $z$ adjacent to two 2 -vertices $x$ and $y$ such that $N(z) \backslash\{x, y\}=$ $\{N(x) \cup N(y)\} \backslash\{z\}$ 
4. $G$ has a 3-vertex $w$ with $N(w)=\{x, y, z\}$ such that both $x$ and $y$ are 2vertices, $N(x)=\{z, w\}$ and $y z \notin E(G)$;

5. $G$ has two adjacent 3-vertices $x$ and $y$ such that $N(x) \cap N(y)=\{z\}$ and $N(z)=\{x, y\}$

6. $G$ has two adjacent 3 -vertices $w_{1}$ and $w_{2}$ such that $N\left(w_{1}\right)=\left\{x, z_{1}, w_{2}\right\}$, $N\left(w_{2}\right)=\left\{y, z_{2}, w_{1}\right\}, N(x)=\left\{z_{1}, w_{1}\right\}$ and $N(y)=\left\{z_{2}, w_{2}\right\} ;$

7. $G$ has a 3-vertex $w$ with $N(w)=\{x, y, z\}$ such that $N(z)=\{w, y\}$ and $x y \in E(G)$;

8. $G$ has two nonadjacent 3 -vertices $w_{1}$ and $w_{2}$ such that $N\left(w_{1}\right)=\left\{x, y, z_{1}\right\}$, $N\left(w_{2}\right)=\left\{x, y, z_{2}\right\}, N\left(z_{1}\right)=\left\{x, w_{1}\right\}$ and $N\left(z_{2}\right)=\left\{y, w_{2}\right\} ;$

9. $G$ has two nonadjacent 3 -vertices $w_{1}$ and $w_{2}$ such that $N\left(w_{1}\right)=\left\{x, y, z_{1}\right\}$, $N\left(w_{2}\right)=\left\{x, y, z_{2}\right\}, N\left(z_{1}\right)=\left\{x, w_{1}\right\}$ and $N\left(z_{2}\right)=\left\{x, w_{2}\right\} ;$

10. $G$ has a 3-vertex $w$ with $N(w)=\left\{x, z_{1}, z_{2}\right\}$ such that there is a 2-vertex $y \in N\left(z_{1}\right) \cap N\left(z_{2}\right)$ and $N(x)=\left\{z_{1}, w\right\}$.

Let $C$ denote color set $\{1,2, \cdots, k\}$. Given a edge-coloring $\varphi$ of $G$ in which $k$ colors in $C$ are used. For each vertex $v \in V(G)$, let $\varphi_{v}^{-1}(i)$ denote the number of edges of $G$ incident with vertex $v$ that receive color $i$ by the coloring $\varphi$, and let $C_{\varphi}(v)=\left\{i \mid \varphi_{v}^{-1}(i)=\min _{1 \leq j \leq k} \varphi_{v}^{-1}(j)\right\}$. Obviously, $\left|C_{\varphi}(v)\right| \geq 1$.

Now we will give the main idea for the proof of our theorem. It is known that a connected graph $G$ has an equitable 2-edge-coloring if and only if it is not an odd circuit. So it is only to prove that any simple and connected SP graph $G$ has an equitable $k$-edge-coloring for any integer $k \geq 3$. We prove the result by induction on $|G|$, the number of vertices of $G$.

When $|G| \leq 4$, the result is obvious. Assume that $p(p \geq 5)$ is an integer and that the theorem holds for all simple SP graphs with less than $p$ vertices. Let $G$ be a simple and connected SP graph of order $p$. If $G$ is not 2-connected, there is a cut vertex $v \in V(G)$ such that $G-v$ is not connected. Select a component of $G-v$ arbitrarily, let $S$ denote its vertex set, $\bar{S}$ denote the set of vertices in $G-v$ which do not belong to $S$. Let $G_{1}$ denote the subgraph of $G$ induced by $S \cup\{v\}, G_{2}$ denote the subgraph of $G$ induced by $\bar{S} \cup\{v\}$. It is obvious that $E(G)=E\left(G_{1}\right) \cup E\left(G_{2}\right)$ and $V(G)=V\left(G_{1}\right) \cup V\left(G_{2}\right)$. Because both $G_{1}$ and $G_{2}$ are SP graphs of order less than $p$, then $G_{1}$ and $G_{2}$ have equitable $k$-edge-colorings $\varphi_{1}$ and $\varphi_{2}$ respectively. Let $k_{1}=k-\left|C_{\varphi_{1}}(v)\right|, k_{2}=\left|C_{\varphi_{2}}(v)\right|$. By interchanging the colors used, an equitable $k$-edge-coloring $\varphi_{1}^{\prime}$ of $G_{1}$ and an equitable $k$-edgecoloring $\varphi_{2}^{\prime}$ of $G_{2}$ will be gained such that $C \backslash C_{\varphi_{1}^{\prime}}(v)=\left\{1,2, \cdots, k_{1}\right\}, C_{\varphi_{2}^{\prime}}(v)=$ $\left\{1,2, \cdots, k_{2}\right\}$. Combine coloring $\varphi_{1}^{\prime}$ in $G_{1}$ with coloring $\varphi_{2}^{\prime}$ in $G_{2}$, an equitable $k$-edge-coloring of $G$ will be gained.

In the following we suppose that $G$ is 2-connected. According to Lemma 1, the proof can be divided into ten parts. In every case as described in Lemma 1, we always obtain a simple SP graph $G^{*}$ of order less than $p$. By the induction hypothesis, $G^{*}$ has an equitable $k$-edge-coloring $\varphi$. On the basis of $\varphi$, we always can construct an equitable $k$-edge-coloring $\sigma$ of $G$ using the same set of $k$ colors. By the induction, the theorem is true. 


\section{Concluding Remarks}

An outerplanar graph is a graph which contains no subgraphs homeomorphic to $K_{4}$ or $K_{2,3}$. That is to say, an outerplanar graph is also a series-parallel graph. From this point of view, our result generalizes the result in [4].

\section{References}

1. Miao lianying, Liu Guizhen: Edge covered coloring and fractional edge covered coloring. J. of Systems Science and Complexing 15(2)(2002) 187-193.

2. D. de Werra: Equitable colorations of graphs. Revue francaise d'Informatique et de Recherche Operationelle, R-3(1971) 3-8.

3. A. J. W. Hilton, D. de Werra: A sufficient condition for equitable edge-coloring of simple graphs. Discrete Math. 128 (1994) 179-201.

4. Wu Jianliang: The equitable edge-coloring of outerplanar graphs. JCMCC 36:1 (2001) 247-253.

5. Wu Jianliang: List edge-coloring of series-parallel graphs. Journal of Shandong University(Natural Science) 35(2) (2000) 144-149.

6. Wu Jianliang: The linear arboricity of series-parallel graphs. Graphs and Combinatorics 16(2000) 367-372. 